\title{
Efficiency of Micro-Stations of Water Treatment in Agroextractivist Projects in the Municipality of Barcarena
}

\author{
Márcio de Freitas Velasco ${ }^{1}$, Davi do Socorro Barros Brasil ${ }^{2}$
}

\begin{abstract}
${ }^{1,2}$ Programa de Pós Graduação em Ciência e Meio Ambiente do Instituto de Ciências Exatas e Naturais da Universidade Federal do Pará (PPGCMA/ICEN/UFPA)
\end{abstract}

Email: marcio.velasco@blm.incra.gov.br, davibb@ufpa.br

Received: January $11^{\text {th }}, 2016$

Accepted: February $14^{\text {th }}, 2016$

Published: Mach 30th 2016

Copyright $@ 2016$ by authors and Institute of Technology Galileo of Amazon (ITEGAM). This work is licensed under the Creative Commons Attribution International License (CC BY 4.0).

http://creativecommons.org/licenses/by/4.0/ c) (1) (8)(2) Open Acces:

\section{ABSTRACT}

The present study allowed to verify the efficiency of the water treatment proposed by the INCRA National Institute of Colonization and Agrarian Reform, to the riverside communities in the Agroextractive Projects Ilha das Onças and Arapiranga Island, in the Municipality of Barcarena, State of Pará. Of treated water and one of raw water for each island, aiming to calculate the efficiency in the treatment through the evaluation of the parameters turbidity, apparent color, residual chlorine and total coliforms. With the results obtained, the good physical quality of the water provided through the low levels of turbidity and apparent color was verified, besides the absence of microbiological contamination and low residual chlorine content, guaranteeing water free of taste and odor. The good quality of the treated water and the high efficiency of the treatment proposed for sources of surface water abstraction can be verified.

Keywords: Water quality. Island of Onças. Arapiranga.

\section{Eficiência de Micro-Estações de Tratamento de Água em Projetos Agroextrativistas no Município de Barcarena}

\begin{abstract}
RESUMO
O presente estudo permitiu verificar a eficiência do tratamento de água proposto pelo INCRA Instituto Nacional de Colonização e Reforma Agrária, às comunidades ribeirinhas nos Projetos Agroextrativistas Ilha das Onças e Ilha Arapiranga, no Município de Barcarena, no Estado do Pará. Foram coletadas 20 amostras de água tratada e uma de água bruta para cada ilha, visando calcular a eficiência no tratamento através da avaliação dos parâmetros turbidez, cor aparente, cloro residual e coliformes totais. Com os resultados obtidos, verificou-se a boa qualidade física da água fornecida através dos baixos níveis de turbidez e cor aparente, além da ausência de contaminação microbiológica e baixo teor de cloro residual, garantindo água isenta de sabor e odor. Pode-se constatar a boa qualidade da água tratada e a elevada eficiência do tratamento proposto para fontes de captação de água superficial.
\end{abstract}

Palavras Chaves: Qualidade da água, Ilha das Onças, Ilha Arapiranga.

\section{INTRODUÇÃO}

De maneira geral a água superficial contém diversos componentes provenientes do próprio ambiente natural, assim como aqueles introduzidos por meio de atividades antropogênicas, sendo que as principais impurezas encontradas em águas superficiais são sólidos dissolvidos na forma ionizada, gases dissolvidos, compostos orgânicos dissolvidos e materiais em suspensão, como microrganismos e colóides, mantendo-se em suspensão estável por longos períodos de tempo, em função das cargas negativas, que 
provocam repulsão entre essas partículas. Tais situações produzem alterações físicas na água, visualmente caracterizadas pelo aumento da turbidez, definida como o grau de redução da passagem de luz pela água, e a presença de cor, causada principalmente pela decomposição de materiais provenientes de resíduos de atividades humanas. Além disso, a água serve como vetor de transmissão de doenças causadas por bactérias, fungos e vírus [6]. São comuns surtos de doenças de veiculação hídrica no meio rural, em virtude do consumo de água sem qualquer tratamento prévio. A ausência de saneamento básico contribui anualmente para a morte de milhares de pessoas, principalmente crianças e idosos, sendo tal situação muito comum na Amazônia [2].

O Programa Territórios da Cidadania foi lançado em 2008, visando romover o desenvolvimento sustentável em áreas de baixo índice

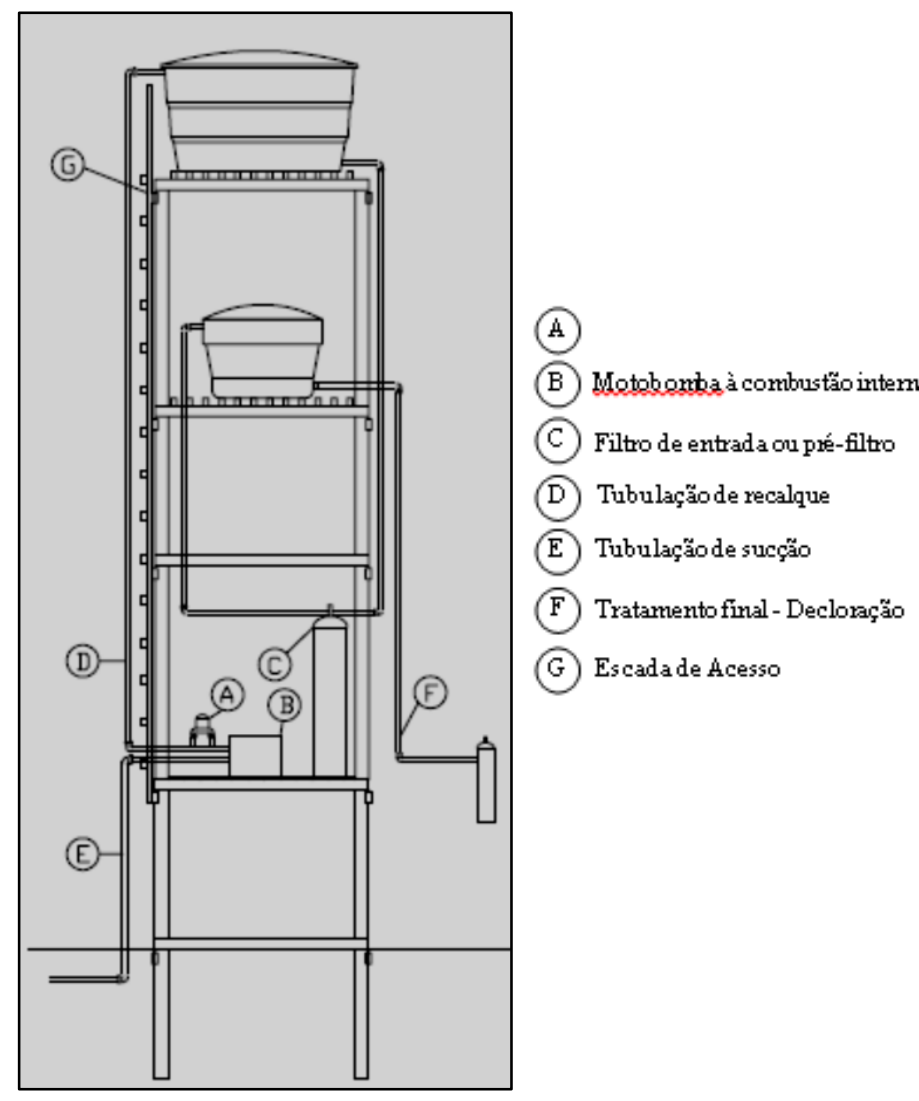

de desenvolvimento humano, tendo como uma de suas diretrizes garantir o acesso ao saneamento e água de qualidade [11]. O Instituto Nacional de Colonização e Reforma Agrária - INCRA, por intermédio do seu corpo técnico na Superintendência Regional em Belém (SR-01), projetou em 2009 um modelo de micro-estação de tratamento de água - META, a fim de garantir às comunidades ribeirinhas água dentro dos padrões de potabilidade estabelecidos pela legislação vigente [3]. A operação de tratamento baseia-se na captação de água de rios e igarapés da região, para ser tratada e distribuída de forma individual ou coletiva, de acordo com a densidade demográfica local, conforme demonstrado nas Figuras 1 e 2 .

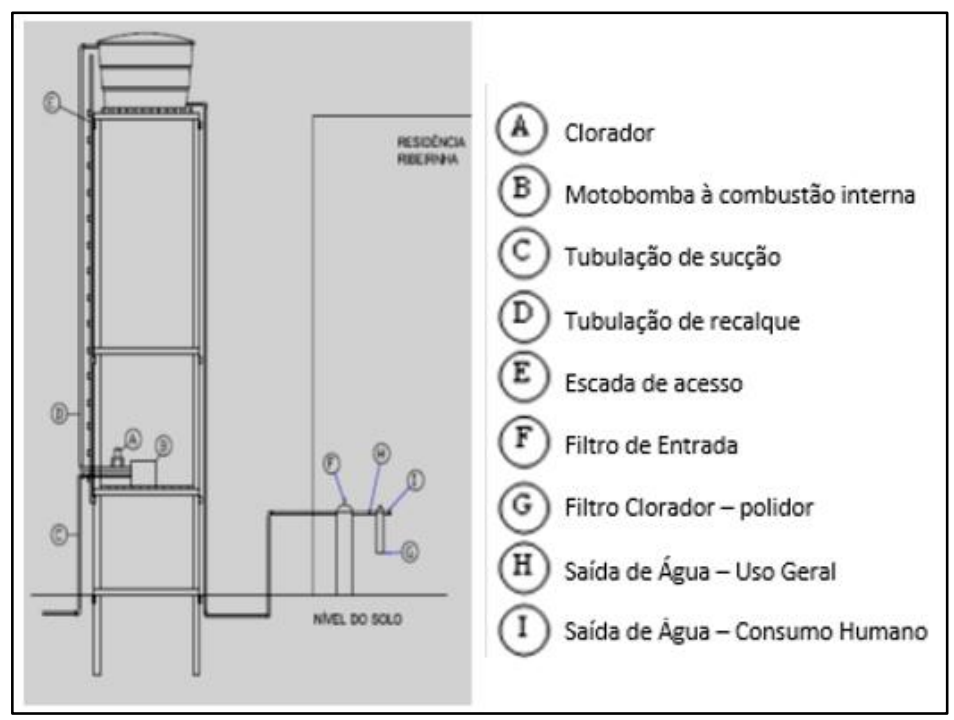

Figura 2: Layout META individual

Fonte: [8].

Figura 1: Layout META coletiva Fonte: [7].

A operação de tratamento de água proposta consiste nas seguintes etapas, conforme demonstrado na Figura 3:

Figura 3: Fluxograma de operação do tratamento de água proposto.

CAPTAÇÃO DE
AGUA BRUTA $\rightarrow \begin{aligned} & \text { CLORAÇÃO }+ \\ & \text { FLOCULAÇAO }\end{aligned} \rightarrow$ FILTRAÇAO $\rightarrow$ POLIMENTO +DECLORAÇAO

Fonte: Os Autores, 2016.

O sistema de tratamento coletivo demonstrado na Figura 1 , consiste basicamente na captação de água bruta diretamente de rios e igarapés, sendo a mesma recalcada para um reservatório elevado, instalado no topo da estrutura de sustentação e armazenamento, em madeira de lei, com 6,40 m de altura. A etapa subseqüente consiste na cloração e floculação, objetivando a desinfecção e clarificação da água bruta, através da implantação de um clorador instalado no barrilete de entrada do reservatório superior, no qual são adicionados ao floculante e o cloro, obedecendo a essa ordem. Em seguida, a água super-clorada descerá por gravidade e passará por um filtro de entrada, localizado na base da estrutura de sustentação, sendo posteriormente 
armazenada em um reservatório intermediário. Em cada residência encontra-se instalada uma central individual de tratamento, para retirada do excesso de cloro residual e matéria em suspensão, garantindo fornecimento de água tratada, de acordo com os padrões estabelecidos pela Portaria $n^{\circ} 2.914$, de 12/12/2011 do Ministério da Saúde [7]. O sistema de tratamento individual demonstrado na Figura 2 mostra praticamente as mesmas características do coletivo, diferenciando-se na altura do reservatório elevado, com 3,00 m de altura, além do fato da água super-clorada descer por gravidade diretamente para residência ribeirinha, onde se encontram instalados em série um filtro de entrada seguido de uma central individual de tratamento, responsáveis pela clarificação, polimento e retirada do excesso de cloro residual, garantindo fornecimento de água tratada, de acordo com os padrões estabelecidos pela Portaria $n^{\circ} 2.914$, de 12/12/2011 do Ministério da Saúde [8].

Os PAE's Ilha das Onças e Ilha Arapiranga, localizados no Município de Barcarena, no Estado do Pará, possuem diversos rios e igarapés, os quais sofrem forte influência das águas da baía do Guajará, que apresentam uma elevada turbidez, são barrentas e de coloração amarelo-esverdeada, situação bem evidente nos seus afluentes, observada principalmente sob maré baixa [5].

Essa pesquisa visa avaliar a eficiência dos sistemas de tratamento de água proposto pelo INCRA através da análise de parâmetros físico-químicos e microbiológicos em amostras de água tratada provenientes de micro-estações de tratamento de água instaladas em residências ribeirinhas nos PAE's Ilha das Onças e Ilha Arapiranga, pertencentes ao Município de Barcarena, no Estado do Pará, tomando-se por base os parâmetros de potabilidade de água estabelecidos pela legislação vigente.

\section{REVISÃO BIBLIOGRÁFICA}

\section{II.1 AMOSTRAGEM.}

Foram coletadas 40 amostras de água tratada em META's instaladas em Projetos Agro-Extrativistas pertencentes ao Município de Barcarena, distribuídas da seguinte forma:

- 20 amostras oriundas de META's coletivas, instaladas no PAE Ilha das Onças, sendo coletada uma amostra de água bruta da fonte de captação comum para todas as amostras de água tratada. Nesse caso, o Igarapé Piramanha. Período de amostragem: 5 a 15 de junho de 2016.

- 20 amostras oriundas de META's individuais, instaladas no PAE Ilha Arapiranga, sendo coletada uma amostra de água bruta da principal fonte de captação, nesse caso o rio Cutajumirim. Período de amostragem: 5 a 15 de setembro de 2016.

O plano de amostragem foi definido em função da localização das META's instaladas em uma mesma drenagem, de acordo com os mapas demonstrados nas Figura 4 e Figura 5. Cada um dos setores teve vinte pontos de amostragem, além de um ponto para coleta de água bruta na drenagem principal. Os parâmetros adotados para avaliação da eficiência do sistema proposto estão diretamente relacionados com as características do manancial superficial utilizado como fonte de captação de água bruta, cuja turbidez e a cor aparente da água a ser tratada apresentam valores elevados, assim como a presença de coliformes totais característica de área sem saneamento básico. A presença ou ausência de cloro residual livre está relacionada com a última etapa do tratamento, a decloração. O protocolo adotado obedeceu a Portaria $n^{\circ} 2.914$, de 12/12/2011 do Ministério da Saúde, que dispõe sobre os procedimentos de controle e de vigilância da qualidade da água para consumo humano e seu padrão de potabilidade [4].

A partir dos resultados obtidos fez-se a comparação entre as duas modalidades propostas: META's coletivas, que atendem até sete famílias ribeirinhas, assim como as META's individuais.

Os mapas de localização dos pontos de amostragem são demonstrados nas Figuras 4 e 5, os quais foram construídos com auxílio do software GPS TrackMaker 13.8, utilizado para georeferenciar e identificar os pontos de amostragem para, em seguida, finalizar a geração dos mapas com auxílio do software QGIS 2.4.

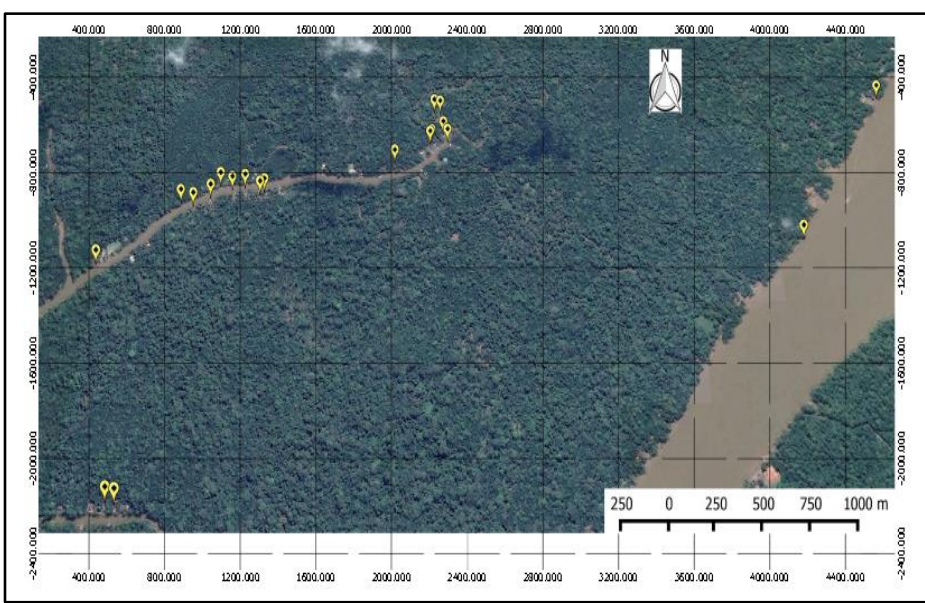

Figura 4: Mapa de amostragem META's individuais (Ilha Arapiranga. Fonte: Os Autores, 2016.

Figura 5: Mapa de amostragem META's coletivas (Ilha da Onças)

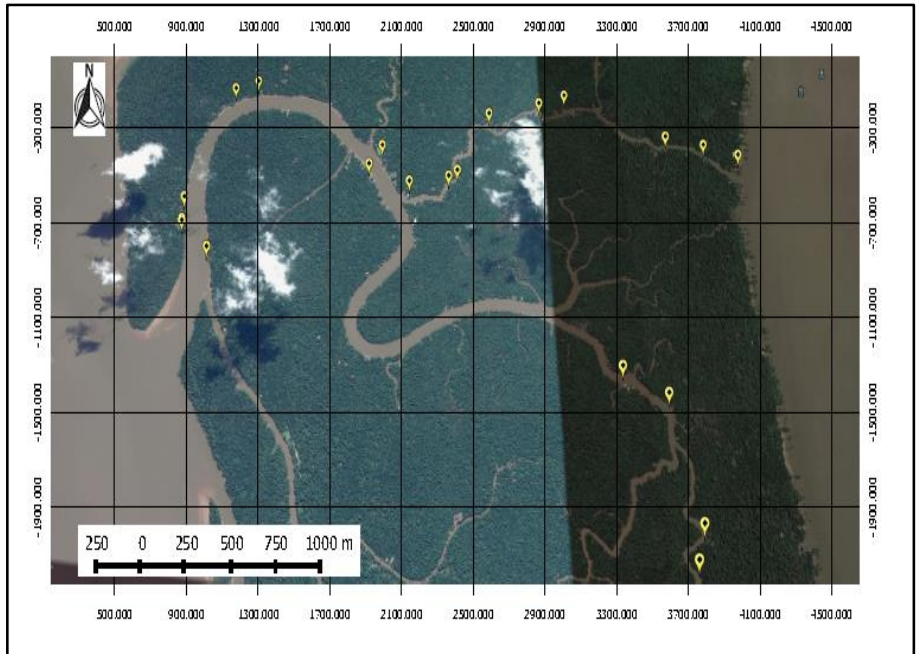

Fonte: Os autores, 2016.

\section{II.2 AVALIAÇÃO DA EFICIÊNCIA DO TRATAMENTO DE ÁGUA PROPOSTO}

As amostras coletadas foram analisadas em laboratório contratado, Monitora Laboratórios LTDA-ME, levando-se em conta que o tratamento de água proposto segue as metodologias convencionais da maioria dos processos adotados pelas companhias de abastecimento de água em cidades espalhadas por 
todo território nacional, que consiste na clarificação e desinfecção da água bruta, sendo que os parâmetros definidos para avaliação da eficiência obedeceram a critérios físicos e microbiológicos determinantes para aceitação do produto final da operação:

turbidez, através do método SMWW 22 Ed., 2130B 2012; cor aparente, através do método SMWW $22^{\circ}$ Ed., $2012-2120 \mathrm{C}$ e coliformes totais, através do método SMWW $22^{\circ}$ Ed., $2012-9221$ $\mathrm{D}$; assim como cloro livre residual, através do método SMWW $22^{\circ}$ Ed., 2012 - $4500 \mathrm{Cl}$, uma vez que a água tratada fornecida será utilizada para consumo humano [1]. Os resultados obtidos foram então comparados com os padrões de potabilidade estabelecidos na legislação vigente. Posteriormente, foi calculada a eficiência no tratamento para cada um dos pontos de coleta. Em seguida, calculou-se a eficiência média para o sistema individual e coletivo. Para a determinação da eficiência no tratamento em relação a cada parâmetro avaliado, utilizou-se a seguinte relação:

Eficiência (\%) = [(valor água bruta - valor água tratada) / valor água bruta] x 100

\section{MATERIAIS E MÉTODOS}

\section{III.1- MICRO-ESTAÇÕES DE TRATAMENTO DE ÁGUA COLETIVAS.}

\section{III.1.1- EFICIÊNCIA NA REMOÇÃO DE TURBIDEZ.}

Através dos resultados obtidos, foi determinada a eficiência na remoção de turbidez da água bruta nas META's coletivas, cujos resultados são apresentados na Tabela 1:

Tabela 1: Resultados para eficiência na remoção de turbidez nas META's coletivas - PAE Ilha das Onças

\begin{tabular}{|c|c|c|c|}
\hline AMOSTRA & $\begin{array}{c}\text { TURBIDEZ } \\
\text { AT (uT) }\end{array}$ & $\begin{array}{c}\text { TURBIDEZ } \\
\text { AB (uT) }\end{array}$ & $\begin{array}{l}\text { EFICIÊNCIA NO } \\
\text { TRATAMENTO }\end{array}$ \\
\hline ON1 & 0,00 & 178,00 & $100,00 \%$ \\
\hline ON2 & 0,00 & 178,00 & $100,00 \%$ \\
\hline ON3 & 0,00 & 178,00 & $100,00 \%$ \\
\hline ON4 & 0,00 & 178,00 & $100,00 \%$ \\
\hline ON5 & 0,00 & 178,00 & $100,00 \%$ \\
\hline ON6 & 0,00 & 178,00 & $100,00 \%$ \\
\hline ON7 & 0,00 & 178,00 & $100,00 \%$ \\
\hline ON8 & 2,00 & 178,00 & $98,88 \%$ \\
\hline ON9 & 1,00 & 178,00 & $99,44 \%$ \\
\hline ON10 & 0,00 & 178,00 & $100,00 \%$ \\
\hline ON11 & 2,00 & 178,00 & $98,88 \%$ \\
\hline ON12 & 1,00 & 178,00 & $99,44 \%$ \\
\hline ON13 & 2,00 & 178,00 & $98,88 \%$ \\
\hline ON14 & 0,00 & 178,00 & $100,00 \%$ \\
\hline ON15 & 0,00 & 178,00 & $100,00 \%$ \\
\hline ON16 & 2,00 & 178,00 & $98,88 \%$ \\
\hline ON17 & 0,00 & 178,00 & $100,00 \%$ \\
\hline ON18 & 1,00 & 178,00 & $99,44 \%$ \\
\hline ON19 & 0,00 & 178,00 & $100,00 \%$ \\
\hline ON20 & 0,00 & 178,00 & $100,00 \%$ \\
\hline & \multicolumn{2}{|c|}{ EFICIÊNCIA MÉDIA } & $99,69 \%$ \\
\hline & \multicolumn{2}{|c|}{$\begin{array}{l}\text { TURBIDEZ MÉDIA ÁGUA } \\
\text { TRATADA }\end{array}$} & 0,55 \\
\hline
\end{tabular}

Fonte: Os autores, 2016.

\section{II.1.2 EFICIÊNCIA NA REMOÇÃO DE COR APARENTE}

A Tabela 2 apresenta os dados relativos a eficiência na remoção de cor aparente.

Tabela 2: Eficiência na remoção de cor aparente.

\begin{tabular}{|c|c|c|c|}
\hline AMOSTRA & $\begin{array}{c}\text { CLORO } \\
\text { RESIDUAL } \\
(\mathrm{mg} / \mathrm{l})\end{array}$ & $\begin{array}{c}\text { CLORO } \\
\text { ADICIONADO } \\
(\mathrm{mg} / \mathrm{l})\end{array}$ & $\begin{array}{l}\text { EFICIENNCIA NO } \\
\text { TRATAMENTO }\end{array}$ \\
\hline ON1 & 0,00 & 2,50 & $100,00 \%$ \\
\hline ON2 & 0,01 & 2,50 & $99,60 \%$ \\
\hline ON3 & 0,00 & 2,50 & $100,00 \%$ \\
\hline ON4 & 0,00 & 2,50 & $100,00 \%$ \\
\hline ON5 & 0,00 & 2,50 & $100,00 \%$ \\
\hline ON6 & 0,00 & 2,50 & $100,00 \%$ \\
\hline ON7 & 0,00 & 2,50 & $100,00 \%$ \\
\hline ON8 & 0,00 & 2,50 & $100,00 \%$ \\
\hline ON9 & 0,00 & 2,50 & $100,00 \%$ \\
\hline ON10 & 0,00 & 2,50 & $100,00 \%$ \\
\hline ON11 & 0,00 & 2,50 & $100,00 \%$ \\
\hline ON12 & 0,00 & 2,50 & $100,00 \%$ \\
\hline ON13 & 0,00 & 2,50 & $100,00 \%$ \\
\hline ON14 & 0,00 & 2,50 & $100,00 \%$ \\
\hline ON15 & 0,00 & 2,50 & $100,00 \%$ \\
\hline ON16 & 0,00 & 2,50 & $100,00 \%$ \\
\hline ON17 & 0,00 & 2,50 & $100,00 \%$ \\
\hline ON18 & 0,00 & 2,50 & $100,00 \%$ \\
\hline ON19 & 0,00 & 2,50 & $100,00 \%$ \\
\hline ON20 & 0,00 & 2,50 & $100,00 \%$ \\
\hline & \multicolumn{2}{|c|}{ EFICIÊNCIA MÉDIA } & $99,98 \%$ \\
\hline & \multicolumn{2}{|c|}{$\begin{array}{c}\text { TEOR MÉDIO DE CLORO } \\
\text { RESIDUAL NA ÁGUA TRATADA }\end{array}$} & 0,00 \\
\hline
\end{tabular}

Fonte: Os autores, 2016.

Por meio dos resultados obtidos, foi determinada a eficiência na remoção de cor aparente da água bruta nas META's coletivas, cujos resultados são apresentados na Tabela 3:

Tabela 3: Resultados para eficiência na remoção de cor aparente nas META's coletivas - PAE Ilha das Onças.

\begin{tabular}{|c|c|c|c|}
\hline AMOSTRA & $\begin{array}{c}\text { COR } \\
\text { APARENTE } \\
\text { AT (UH) }\end{array}$ & $\begin{array}{c}\text { COR } \\
\text { APARENTE } \\
\text { AB (UH) }\end{array}$ & $\begin{array}{l}\text { EFICIÊNCIA NO } \\
\text { TRATAMENTO }\end{array}$ \\
\hline ON1 & 1,00 & 32,00 & $96,88 \%$ \\
\hline ON2 & 1,00 & 32,00 & $96,88 \%$ \\
\hline ON3 & 1,00 & 32,00 & $96,88 \%$ \\
\hline ON4 & 1,00 & 32,00 & $96,88 \%$ \\
\hline ON5 & 0,00 & 32,00 & $100,00 \%$ \\
\hline ON6 & 0,00 & 32,00 & $100,00 \%$ \\
\hline ON7 & 0,00 & 32,00 & $100,00 \%$ \\
\hline ON8 & 1,00 & 32,00 & $96,88 \%$ \\
\hline ON9 & 5,00 & 32,00 & $84,38 \%$ \\
\hline ON10 & 0,00 & 32,00 & $100,00 \%$ \\
\hline ON11 & 1,00 & 32,00 & $96,88 \%$ \\
\hline ON12 & 2,00 & 32,00 & $93,75 \%$ \\
\hline ON13 & 6,00 & 32,00 & $81,25 \%$ \\
\hline ON14 & 6,00 & 32,00 & $81,25 \%$ \\
\hline ON15 & 4,00 & 32,00 & $87,50 \%$ \\
\hline ON16 & 6,00 & 32,00 & $81,25 \%$ \\
\hline ON17 & 0,00 & 32,00 & $100,00 \%$ \\
\hline ON18 & 5,00 & 32,00 & $84,38 \%$ \\
\hline ON19 & 0,00 & 32,00 & $100,00 \%$ \\
\hline ON2O & 0,00 & 32,00 & $100,00 \%$ \\
\hline & \multicolumn{2}{|c|}{ EFICIÊNCIA MÉDIA } & $93,75 \%$ \\
\hline & \multicolumn{2}{|c|}{$\begin{array}{c}\text { COR APARENTE MÉDIA } \\
\text { ÁGUA TRATADA }\end{array}$} & 2,00 \\
\hline
\end{tabular}

Fonte: Os autores, 2016. 


\section{III.1.3- EFICIÊNCIA NA DECLORAÇÃO E EFICIÊNCIA NA DESINFECÇÃO DA ÁGUA BRUTA}

$\mathrm{Na}$ operação de cloração efetuada na etapa inicial são adicionados $2,5 \mathrm{mg} / \mathrm{l}$ de cloro ativo para desinfecção da água bruta, sendo efetuada a decloração na etapa final do processo, gerando assim os dados demonstrados na Tabela 4, comprovando a eficiência nessa etapa de tratamento:

Tabela 4: Resultados para eficiência da decloração nas META's coletivas - PAE Ilha das Onças.

\begin{tabular}{|c|c|c|c|}
\hline AMOSTRA & $\begin{array}{c}\text { COLIFORMES } \\
\text { TOTAIS AT } \\
\text { (P-A/100 mI) }\end{array}$ & $\begin{array}{c}\text { COLIFORMES } \\
\text { TOTAIS AB } \\
\text { (P-A/100 mI) }\end{array}$ & $\begin{array}{c}\text { EFICIENCIA NO } \\
\text { TRATAMENTO }\end{array}$ \\
\hline ON1 & AUSENTE & PRESENÇA & $100,00 \%$ \\
\hline ON2 & AUSENTE & PRESENÇA & $100,00 \%$ \\
\hline ON3 & AUSENTE & PRESENÇA & $100,00 \%$ \\
\hline ON4 & AUSENTE & PRESENÇA & $100,00 \%$ \\
\hline ON5 & AUSENTE & PRESENÇA & $100,00 \%$ \\
\hline ON6 & AUSENTE & PRESENÇA & $100,00 \%$ \\
\hline ON7 & AUSENTE & PRESENÇA & $100,00 \%$ \\
\hline ON8 & AUSENTE & PRESENÇA & $100,00 \%$ \\
\hline ON9 & AUSENTE & PRESENÇA & $100,00 \%$ \\
\hline ON10 & AUSENTE & PRESENÇA & $100,00 \%$ \\
\hline ON11 & AUSENTE & PRESENÇA & $100,00 \%$ \\
\hline ON12 & AUSENTE & PRESENÇA & $100,00 \%$ \\
\hline ON13 & AUSENTE & PRESENÇA & $100,00 \%$ \\
\hline ON14 & AUSENTE & PRESENÇA & $100,00 \%$ \\
\hline ON15 & AUSENTE & PRESENÇA & $100,00 \%$ \\
\hline ON16 & AUSENTE & PRESENÇA & $100,00 \%$ \\
\hline ON17 & AUSENTE & PRESENÇA & $100,00 \%$ \\
\hline ON18 & AUSENTE & PRESENÇA & $100,00 \%$ \\
\hline ON19 & AUSENTE & PRESENÇA & $100,00 \%$ \\
\hline ON20 & AUSENTE & PRESENÇA & $100,00 \%$ \\
\hline & \multicolumn{2}{|c|}{ EFICIENCIA MÉDIA } & $100,00 \%$ \\
\hline
\end{tabular}

Fonte: Os autores, 2016.

\section{III.2 MICRO-ESTAÇÃO DE TRATAMENTO DE ÁGUA INDIVIDUAL E A EFICIÊNCIA NA REMOÇÃO DE TURBIDEZ}

Através dos resultados obtidos, foi determinada a eficiência na remoção de turbidez da água bruta nas META's individuais, cujos resultados são apresentados na Tabela 5:

Tabela 5: Resultados para eficiência na remoção de turbidez nas META's individuais - PAE Ilha Arapiranga.

\begin{tabular}{|c|c|c|c|}
\hline AMOSTRA & $\begin{array}{l}\text { TURBIDEZ } \\
\text { AT (uT) }\end{array}$ & $\begin{array}{l}\text { TURBIDEZ } \\
\text { AB (UT) }\end{array}$ & $\begin{array}{l}\text { EFICIÊNCIA NO } \\
\text { TRATAMENTO }\end{array}$ \\
\hline AR1 & 0,00 & 146,00 & $100,00 \%$ \\
\hline AR2 & 0,00 & 146,00 & $100,00 \%$ \\
\hline AR3 & 0,00 & 146,00 & $100,00 \%$ \\
\hline AR4 & 0,00 & 146,00 & $100,00 \%$ \\
\hline AR5 & 0,00 & 146,00 & $100,00 \%$ \\
\hline AR6 & 0,00 & 146,00 & $100,00 \%$ \\
\hline AR7 & 0,00 & 146,00 & $100,00 \%$ \\
\hline AR8 & 0,00 & 146,00 & $100,00 \%$ \\
\hline AR9 & 0,00 & 146,00 & $100,00 \%$ \\
\hline AR10 & 0,00 & 146,00 & $100,00 \%$ \\
\hline AR11 & 0,00 & 146,00 & $100,00 \%$ \\
\hline AR12 & 0,00 & 146,00 & $100,00 \%$ \\
\hline AR13 & 0,00 & 146,00 & $100,00 \%$ \\
\hline AR14 & 0,00 & 146,00 & $100,00 \%$ \\
\hline AR15 & 0,00 & 146,00 & $100,00 \%$ \\
\hline AR16 & 0,00 & 146,00 & $100,00 \%$ \\
\hline AR17 & 0,00 & 146,00 & $100,00 \%$ \\
\hline AR18 & 0,00 & 146,00 & $100,00 \%$ \\
\hline AR19 & 0,00 & 146,00 & $100,00 \%$ \\
\hline AR20 & 0,00 & 146,00 & $100,00 \%$ \\
\hline & \multicolumn{2}{|c|}{ EFICIÊNCIA MÉDIA } & $100,00 \%$ \\
\hline & \multicolumn{2}{|c|}{ TURBIDEZ MÉDIA ÁGUA } & 0,00 \\
\hline
\end{tabular}

Fonte: Os autores, 2016.

\section{III.2.2- EFICIÊNCIA NA REMOÇÃO DE COR APARENTE}

Por meio dos resultados obtidos, foi determinada a eficiência na remoção de cor aparente da água bruta nas META's individuais, cujos resultados são apresentados na Tabela 6:

Tabela 6: Resultados para eficiência na remoção de cor aparente nas META's individuais - PAE Ilha Arapiranga.

\begin{tabular}{|c|c|c|c|}
\hline AMOSTRA & $\begin{array}{c}\text { COR } \\
\text { APARENTE } \\
\text { AT (uH) } \\
\end{array}$ & $\begin{array}{c}\text { COR } \\
\text { APARENTE } \\
\text { AB (uH) } \\
\end{array}$ & $\begin{array}{l}\text { EFICIÊNCIA NO } \\
\text { TRATAMENTO }\end{array}$ \\
\hline AR1 & 0,00 & 25,00 & $100,00 \%$ \\
\hline AR2 & 0,00 & 25,00 & $100,00 \%$ \\
\hline AR3 & 0,00 & 25,00 & $100,00 \%$ \\
\hline AR4 & 0,00 & 25,00 & $100,00 \%$ \\
\hline AR5 & 0,00 & 25,00 & $100,00 \%$ \\
\hline AR6 & 0,00 & 25,00 & $100,00 \%$ \\
\hline AR7 & 0,00 & 25,00 & $100,00 \%$ \\
\hline AR8 & 0,00 & 25,00 & $100,00 \%$ \\
\hline AR9 & 0,00 & 25,00 & $100,00 \%$ \\
\hline AR10 & 0,00 & 25,00 & $100,00 \%$ \\
\hline AR11 & 0,00 & 25,00 & $100,00 \%$ \\
\hline AR12 & 0,00 & 25,00 & $100,00 \%$ \\
\hline AR13 & 0,00 & 25,00 & $100,00 \%$ \\
\hline AR14 & 0,00 & 25,00 & $100,00 \%$ \\
\hline AR15 & 0,00 & 25,00 & $100,00 \%$ \\
\hline AR16 & 0,00 & 25,00 & $100,00 \%$ \\
\hline AR17 & 0,00 & 25,00 & $100,00 \%$ \\
\hline AR18 & 0,00 & 25,00 & $100,00 \%$ \\
\hline AR19 & 0,00 & 25,00 & $100,00 \%$ \\
\hline AR20 & 0,00 & 25,00 & $100,00 \%$ \\
\hline & \multicolumn{2}{|c|}{ EFICIÉNCIA MÉDIA } & $100,00 \%$ \\
\hline & \multicolumn{2}{|c|}{$\begin{array}{c}\text { COR APARENTE MÉDIA } \\
\text { ÁGUA TRATADA }\end{array}$} & 0,00 \\
\hline
\end{tabular}

Fonte: Os autores, 2016.

\section{III.2.3- EFICIÊNCIA NA DECLORAÇÃO}

Na operação de cloração efetuada na etapa inicial são adicionados 2,5 mg/l de cloro ativo para desinfecção da água bruta, sendo efetuada a decloração na etapa final do processo, gerando assim os dados demonstrados na Tabela 7, comprovando a eficiência nessa etapa de tratamento:

Quadro 7 - Resultados para eficiência da decloração nas META's individuais - PAE Ilha Arapiranga

\begin{tabular}{|c|c|c|c|}
\hline AMOSTRA & $\begin{array}{c}\text { CLORO } \\
\text { RESIDUAL } \\
(\mathrm{mg} / \mathrm{l})\end{array}$ & $\begin{array}{c}\text { CLORO } \\
\text { ADICIONADO } \\
(\mathrm{mg} / \mathrm{l})\end{array}$ & $\begin{array}{l}\text { EFICIÊNCIA NO } \\
\text { TRATAMENTO }\end{array}$ \\
\hline AR1 & 0,00 & 2,50 & $100,00 \%$ \\
\hline AR2 & 0,00 & 2,50 & $100,00 \%$ \\
\hline AR3 & 0,00 & 2,50 & $100,00 \%$ \\
\hline AR4 & 0,00 & 2,50 & $100,00 \%$ \\
\hline AR5 & 0,00 & 2,50 & $100,00 \%$ \\
\hline AR6 & 0,00 & 2,50 & $100,00 \%$ \\
\hline AR7 & 0,00 & 2,50 & $100,00 \%$ \\
\hline AR8 & 0,00 & 2,50 & $100,00 \%$ \\
\hline AR9 & 0,00 & 2,50 & $100,00 \%$ \\
\hline AR10 & 0,00 & 2,50 & $100,00 \%$ \\
\hline AR11 & 0,00 & 2,50 & $100,00 \%$ \\
\hline AR12 & 0,00 & 2,50 & $100,00 \%$ \\
\hline AR13 & 0,00 & 2,50 & $100,00 \%$ \\
\hline AR14 & 0,00 & 2,50 & $100,00 \%$ \\
\hline AR15 & 0,00 & 2,50 & $100,00 \%$ \\
\hline AR16 & 0,00 & 2,50 & $100,00 \%$ \\
\hline AR17 & 0,00 & 2,50 & $100,00 \%$ \\
\hline AR18 & 0,00 & 2,50 & $100,00 \%$ \\
\hline AR19 & 0,00 & 2,50 & $100,00 \%$ \\
\hline AR2O & 0,00 & 2,50 & $100,00 \%$ \\
\hline & \multicolumn{2}{|c|}{ EFICIÊNCIA MÉDIA } & $100,00 \%$ \\
\hline & \multicolumn{2}{|c|}{ TEOR MÉDIO DE CLORO } & 0,00 \\
\hline
\end{tabular}

Fonte: Os autores, 2016. 


\section{III.2.4 EFICIÊNCIA NA DESINFECÇÃO DA ÁGUA BRUTA}

Na operação de cloração efetuada na etapa inicial são adicionados $2,5 \mathrm{mg} / 1$ de cloro ativo para desinfecção da água bruta, gerando assim os dados demonstrados na Tabela 8, comprovando a eficiência nessa etapa de tratamento:

Tabela 8: Resultados para eficiência da desinfecção da água bruta nas META's individuais - PAE Ilha Arapiranga.

\begin{tabular}{|c|c|c|c|}
\hline AMOSTRA & $\begin{array}{c}\text { COLIFORMES } \\
\text { TOTAIS AT } \\
\text { (P-A/100 mI) }\end{array}$ & $\begin{array}{c}\text { COLIFORMES } \\
\text { TOTAIS AB } \\
\text { (P-A/100 ml) }\end{array}$ & $\begin{array}{c}\text { EFICIÊNCIA NO } \\
\text { TRATAMENTO }\end{array}$ \\
\hline AR1 & AUSENTE & PRESENÇA & $100,00 \%$ \\
\hline AR2 & AUSENTE & PRESENÇA & $100,00 \%$ \\
\hline AR3 & AUSENTE & PRESENÇA & $100,00 \%$ \\
\hline AR4 & AUSENTE & PRESENÇA & $100,00 \%$ \\
\hline AR5 & AUSENTE & PRESENÇA & $100,00 \%$ \\
\hline AR6 & AUSENTE & PRESENÇA & $100,00 \%$ \\
\hline AR7 & AUSENTE & PRESENÇA & $100,00 \%$ \\
\hline AR8 & AUSENTE & PRESENÇA & $100,00 \%$ \\
\hline AR9 & AUSENTE & PRESENÇA & $100,00 \%$ \\
\hline AR10 & AUSENTE & PRESENÇA & $100,00 \%$ \\
\hline AR11 & AUSENTE & PRESENÇA & $100,00 \%$ \\
\hline AR12 & AUSENTE & PRESENÇA & $100,00 \%$ \\
\hline AR13 & AUSENTE & PRESENÇA & $100,00 \%$ \\
\hline AR14 & AUSENTE & PRESENÇA & $100,00 \%$ \\
\hline AR15 & AUSENTE & PRESENÇA & $100,00 \%$ \\
\hline AR16 & AUSENTE & PRESENÇA & $100,00 \%$ \\
\hline AR17 & AUSENTE & PRESENÇA & $100,00 \%$ \\
\hline AR18 & AUSENTE & PRESENÇA & $100,00 \%$ \\
\hline AR19 & AUSENTE & PRESENÇA & $100,00 \%$ \\
\hline AR20 & AUSENTE & PRESENÇA & $100,00 \%$ \\
\cline { 2 - 4 } & & & $100,00 \%$ \\
\hline \multirow{2}{*}{ ERFICIÊNCIA MÉDIA } & \\
\hline Fon & & & \\
\hline
\end{tabular}

Fonte: Os autores, 2016.

Os resultados demonstram que ambos os sistemas apresentam elevada eficiência para cada parâmetro avaliado. $\mathrm{O}$ sistema coletivo apresentou eficiência levemente inferior para os parâmetros cor aparente e turbidez em algumas amostras. Essa situação pode estar relacionada a determinados fatores. As META's coletivas foram instaladas no PAE Ilha das Onças no ano de 2014, tendo maior tempo de funcionamento, fato gerador da necessidade de manutenção nos sistemas. $\mathrm{O}$ volume de reserva é superior a 2.000 litros, necessita de uma quantidade maior de reagente e maior tempo reacional para atingir o máximo de eficiência no tratamento da água bruta. Já as META's individuais apresentam um menor volume de reserva, 500 litros e necessitam de menor quantidade de reagentes e menor tempo reacional, além de apresentarem menor custo de instalação. Outro fato importante reside no fato dos sistemas individuais terem sido instalados no ano de 2016.

\section{RESULTADOS E DISCUSSÕES}

As duas variedades do sistema de tratamento de água proposto pelo INCRA avaliadas distinguem-se de forma sutil quanto ao nível de eficiência para os parâmetros turbidez e cor aparente, mas ambos apresentam máxima eficiência na operação de desinfecção da água bruta. A decloração e o polimento da água tratada na etapa final do processo garante um produto final com propriedades organolépticas apreciáveis para o consumo humano, sendo essa característica muito importante para sua aceitação por parte dos principais interessados, os ribeirinhos.
Ficou evidenciada a necessidade de um acompanhamento mais contundente das unidades já instaladas a fim de garantir a máxima eficiência no processo de obtenção de água tratada dentro dos padrões de potabilidade exigidos pela legislação vigente. De forma geral, a água fornecida pelas micros estações de tratamento de água projetadas pelo INCRA utilizada para consumo doméstico nas comunidades ribeirinhas residentes nos PAE's Ilha das Onças e Ilha Arapiranga apresenta boa qualidade física evidenciada pelos baixos níveis de turbidez e cor aparente, com valores médios de $0,55 \mathrm{uT}$ e 2,00 uH respectivamente para modalidade coletiva; assim como $0,00 \mathrm{uT}$ e $0,00 \mathrm{uH}$ respectivamente para a modalidade individual. A ausência de contaminação microbiológica evidenciada nos resultados poderá colaborar com a redução nos casos de doenças de veiculação hídrica, tão comuns no meio rural. Os baixos teores de cloro residual garantem água isenta de sabor e odor que, na maioria das vezes, causa desconfiança no consumo por parte dos ribeirinhos, que tem como parâmetro organoléptico a água mineral.

\section{REFERÊNCIAS BIBLIOGRÁFICAS}

BRITTO, Fábio Brandão et al. Monitoramento e modelagem da qualidade da água e agrotóxicos em corpos hídricos no Baixo São Francisco sergipano. 2015.

DA SILVA, Eunice Ferreira; NACHORNIK, Valdomiro Lourenço. Ação Cívico-Social (ACiSo): A experiência de estudantes universitários participantes do Projeto Rondon na Ilha do Marajó, Estado do Pará. Revista ELO-Diálogos em Extensão, v. 4. n. 1, 2015.

\section{INCRA, 2015. RELATÓRIO DE GESTÃO DO EXERCÍCIO DE 2014. Disponível em} http://www.incra.gov.br/tree/info/file/8868. Acesso em 30 Set. 2016.

MINISTÉRIO DA SAÚDE. Portaria $\mathbf{n}^{\circ} \mathbf{2 . 9 1 4}$, de 12 de dezembro de 2011. Disponível em http://bvsms.saude.gov.br/bvs/saudelegis/gm/2011/prt2914_12_1 2_2011.html. Acesso em 30 Set. 2016.

MOURA, Elyana Melo. Mapeamento do halo de dispersão formado por efluentes industriais lançados na baía do Guajará no trecho compreendido entre o bairro de Val-de-Cães e o distrito de Icoaraci. Programa de Pós-graduação em Geologia e Geoquímica. Universidade Federal do Pará, Centro de Geociências, Belém-PA, 2007.

PAVANELLI, Gerson. Eficiência de diferentes tipos de coagulantes na coagulação, floculação e sedimentação de água com cor ou turbidez elevada. 2001. Tese de Doutorado. Universidade de São Paulo.

PORTAL DOS CONVÊNIOS, 2013. Convênio no 791882/2013. Disponível em https://www.convenios.gov.br/siconv/ConsultarProposta/Resultad oDaConsultaDeConvenioSelecionarConvenio.do?idConvenio=37 4402\&destino=. Acesso em 30 Set. 2016.

PORTAL DOS CONVÊNIOS, 2014. Convênio no 802393/2014. Disponível 
https://www.convenios.gov.br/siconv/ConsultarProposta/Resultad oDaConsultaDeConvenioSelecionarConvenio.do?idConvenio $=41$ 1743\&destino=. Acesso em 30 Set. 2016.

RÔLA, Anoar Kayali Koubeissi et al. AVALIAÇÃO DA EFICIÊNCIA DE COAGULANTES COMERCIAIS PARA APLICAÇÃO EM SISTEMAS DE TRATAMENTO DE ÁGUA. Journal JCEC/REQ2, v. 2, n. 3, p. 014-033, 2016.

SOUZA, APC; SOUZA, EAM; PEREIRA, N. C. ANÁLISE DA UTILIZAÇÃO DO COAGULANTE POLICLORETO DE ALUMÍNIO (PAC) NA REMOÇÃO DA COR, TURBIDEZ E DQO DE EFLUENTE DE LAVANDERIA TEXTIL. Blucher Chemical Engineering Proceedings, v. 1, n. 2, p. 9566-9572, 2015.

VISÚ, Gilson Carlos et al. O programa territórios da cidadania: uma análise a partir do cone sul de Mato Grosso do Sul. Observatorio de la Economía Latinoamericana, n. 203, 2014. 